\title{
Large-bore Arterial Access in the Era of Structural Cardiovascular Disease
}

\author{
Jelani Grant ${ }^{1}$, Akash Maniam ${ }^{2}$, Diego Celli ${ }^{1}$, Vicente Orozco-Sevilla ${ }^{3}$, and Joao Braghiroli ${ }^{1}$ \\ ${ }^{1}$ University of Miami Health System \\ ${ }^{2}$ Sangre Grande Hospital \\ ${ }^{3}$ Baylor College of Medicine
}

July 9, 2020

\begin{abstract}
Over the last two decades, the medical community witnessed an outstanding and accelerated development on minimally invasive therapies. With the dorsal spine of supportive data from large randomized control trials, transcatheter aortic valve replacement (TAVR), aortic and mitral valve-in-valve, mechanical circulatory support and peripheral endovascular interventions all share the need of accessing a vascular bed with a large bore catheter. Nevertheless, to date, there has yet to be a universal consensus on defining large-bore vascular access (LBVA) in the world of transcatheter therapies. We explore the evolution, characteristics and vascular compatibility of the current commercially available devices, analyze the devices along with access site-specific complications rates and finally review the present methods for percutaneous vascular closure.
\end{abstract}

\section{Hosted file}

LBVA_Arterial_Maindocument.docx available at https://authorea.com/users/341322/articles/ 468305-large-bore-arterial-access-in-the-era-of-structural-cardiovascular-disease 
Table 1. Large Bore Vascular Devices with Minimal Vessel Size

\begin{tabular}{|c|c|c|c|c|}
\hline Delivery Device & $\begin{array}{l}\text { Vascular } \\
\text { Access } \\
\text { Site } \\
\end{array}$ & Procedure & $\begin{array}{l}\text { Size in } \\
\text { Fr }\end{array}$ & \begin{tabular}{|l|l|}
\multicolumn{2}{|l|}{ Recommended } \\
Minimal & Vessel \\
Size/mm & \\
\end{tabular} \\
\hline Sapien 3 Commander Delivery System & \multirow{11}{*}{$\begin{array}{l}\text { Femoral } \\
\text { Artery }\end{array}$} & \multirow{11}{*}{$\begin{array}{l}\text { Transcatheter } \\
\text { aortic valve } \\
\text { replacement }\end{array}$} & $14-16$ & $5.5-6.0$ \\
\hline Edwards Axela Sheath & & & 14 & $\begin{array}{l}5.5 \quad(6.0 \text { with } \\
\text { subclavian/axillary } \\
\text { access) }\end{array}$ \\
\hline Evolut R/EnVeo R & & & $14-16$ & 5.0 \\
\hline Evolut Pro/Enveo Pro & & & $14-16$ & 5.0 \\
\hline Evolut Pro + & & & $14-18$ & $\begin{array}{l}5.0(5.5 \text { for } 16 \mathrm{Fr} \\
\text { and } 6.0 \text { for } 18 \mathrm{Fr})\end{array}$ \\
\hline Lotus Edge/iSleeve-Lotus Introducer Set & & & 15 & $\begin{array}{l}6.5 \text { with Lotus } \\
\text { Introducer Set/5.9 } \\
\text { with iSleeve }\end{array}$ \\
\hline \multirow[t]{2}{*}{ Acurate Neo Aortic Valve System } & & & & \multirow[b]{2}{*}{5.9 with the } \\
\hline & & & 18 & \\
\hline JenaValve Pericardial TAVR System & & & & $\begin{array}{l}\text { Class III } \\
\text { investigational } \\
\text { device undergoing } \\
\text { clinical trials in the } \\
\text { US (not yet FDA } \\
\text { approved) }\end{array}$ \\
\hline Venus Valve & & & & \\
\hline Allegra Valve & & & 18 & \\
\hline Impella CP & \multirow[t]{3}{*}{$\begin{array}{l}\text { Femoral } \\
\text { Artery }\end{array}$} & \multirow{3}{*}{$\begin{array}{l}\text { Mechanical } \\
\text { Circulatory } \\
\text { Support (Left } \\
\text { Ventricle to } \\
\text { Aorta Assist } \\
\text { Device) }\end{array}$} & $\begin{array}{l}14 \text { (23 } \\
\text { Fr for } \\
\text { axillary } \\
\text { access) }\end{array}$ & \\
\hline Impella 5 & & & 21 & \\
\hline Impella RP & & & 22 & $\begin{array}{l}\text { Body Surface Area } \\
\geq 1.5 \mathrm{~m}^{2}\end{array}$ \\
\hline TandemHeart & $\begin{array}{l}\text { Femoral } \\
\text { Artery }\end{array}$ & $\begin{array}{l}\text { Mechanical } \\
\text { Circulatory } \\
\text { Support (Left } \\
\text { Atrium to } \\
\text { Aorta Assist } \\
\text { Device) }\end{array}$ & & \\
\hline
\end{tabular}


Table 2. Currently Available, FDA-Approved Stent Grafts for Treatment of Thoracic Aortic Pathology.

\begin{tabular}{|l|l|l|l|}
\hline $\begin{array}{l}\text { Product Name } \\
\text { (Manufacturer) }\end{array}$ & $\begin{array}{l}\text { Available Graft } \\
\text { Sizes (mm) }\end{array}$ & $\begin{array}{l}\text { Proximal Neck } \\
\text { Diameter (mm) }\end{array}$ & $\begin{array}{l}\text { Sheath/Introduction } \\
\text { System Diameter (F) }\end{array}$ \\
\hline $\begin{array}{l}\text { TAG } \\
\text { conformable } \\
\text { thoracic } \\
\text { endoprosthesis } \\
\text { (Gore and } \\
\text { Associates }\end{array}$ & $21-45$ & $16-42$ & $\begin{array}{l}18-24 \text { (inner diameter; } \\
\text { sheath required) }\end{array}$ \\
\hline $\begin{array}{l}\text { The RelayPlus } \\
\text { System (Bolton } \\
\text { Medical, Inc.) }\end{array}$ & $22-46$ & $19-42$ & $22-26$ (outer diameter) \\
\hline $\begin{array}{l}\text { Valiant thoracic } \\
\text { stent graft } \\
\text { (Medtronic) }\end{array}$ & $22-46$ & $18-42$ & $22-25$ (outer diameter) \\
\hline $\begin{array}{l}\text { Zenith Alpha } \\
\text { thoracic device } \\
\text { (Cook Medical) }\end{array}$ & $24-46$ & $20-42$ & $16-20$ (inner diameter) \\
\hline Abbreviations: FDA, US Food and Drug Administration \\
\hline
\end{tabular}

Table 3. Examples of Endovascular Aneurysmal Repair Delivery Diameters.

\begin{tabular}{|l|l|l|l|l|}
\hline Device & Manufacturer & $\begin{array}{l}\text { Outer Diameter } \\
(\mathbf{F})^{*}\end{array}$ & $\begin{array}{l}\text { CE Mark } \\
\text { Approval }\end{array}$ & FDA Approval \\
\hline Incraft & $\begin{array}{l}\text { Cordis } \\
\text { Corporation }\end{array}$ & 14 & Yes & No \\
\hline Ovation & Endologix & 14 & Yes & Yes \\
\hline Nellix & Endologix & 17 & Yes & No \\
\hline AFX & Endologix & 17 & Yes & Yes \\
\hline $\begin{array}{l}\text { Zenith Alpha } \\
\text { AAA }\end{array}$ & Cook Medical & 18 & Yes & No \\
\hline Endurant II & Medtronic & 18 & Yes & Yes \\
\hline Excluder & $\begin{array}{l}\text { Gore \& } \\
\text { Associates }\end{array}$ & $20.4 \dagger$ & Yes & Yes \\
\hline $\begin{array}{l}\text { Abbreviations: CE, Conformité Européenne; FDA, US Food and Drug Administration } \\
\text { * Size represents the majority of the main body devices in the product range. } \\
+ \text { Outer diameter of 18-F introducer sheath. }\end{array}$ \\
\hline
\end{tabular}



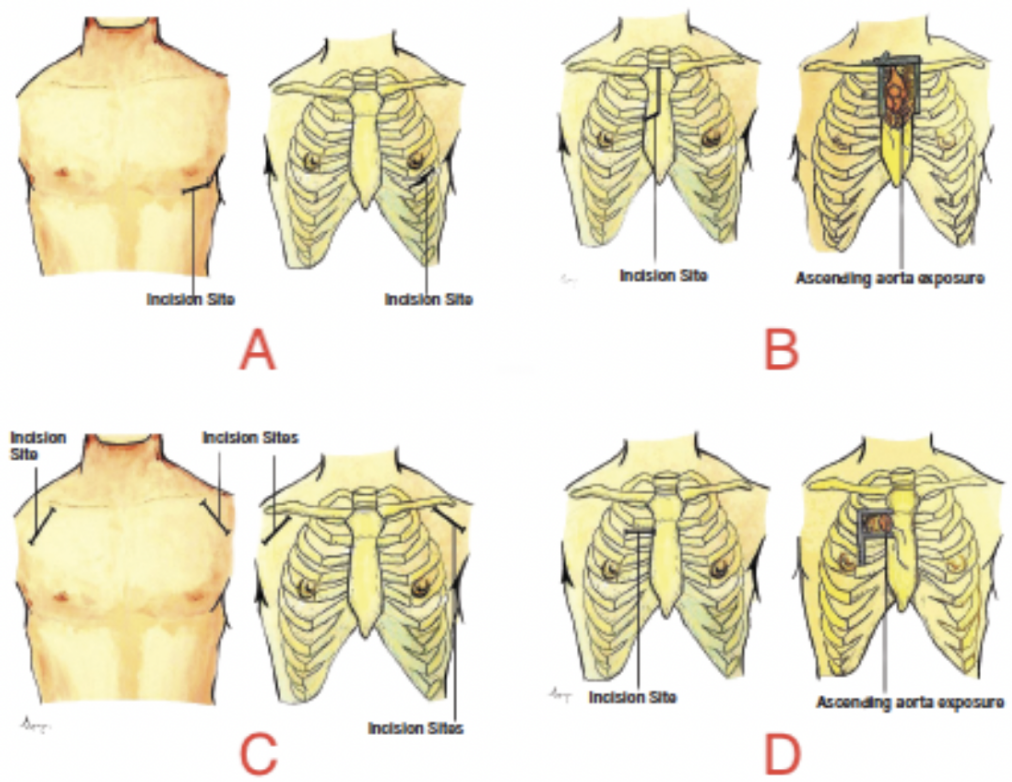


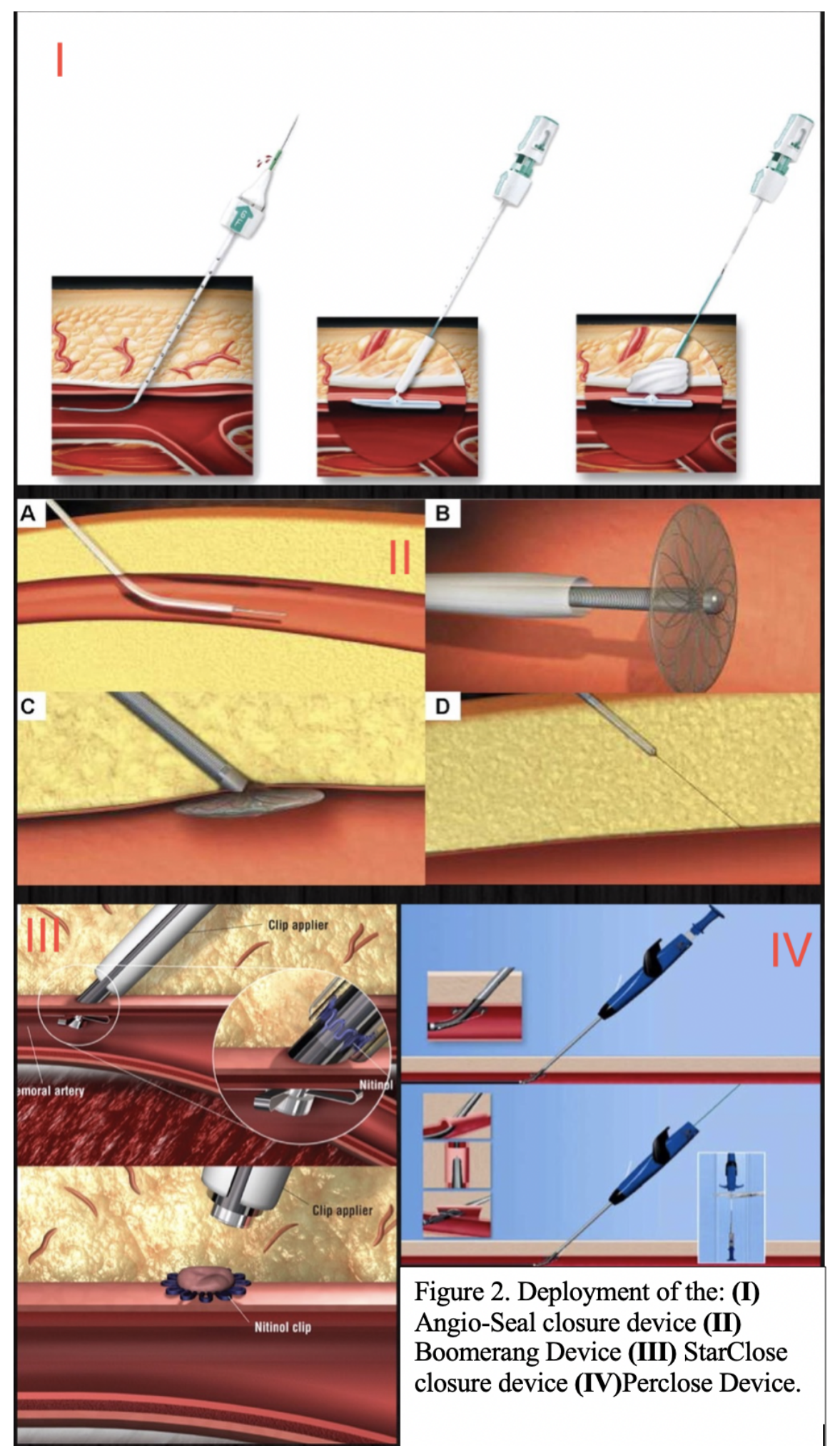

